OPEN ACCESS

Edited by:

Qi Chao,

Deakin University, Australia

Reviewed by:

Qichi Le,

Northeastern University, China

Xin Zhang,

Hebei University of Technology, China

Tingting Guo,

Xi'an Technological University, China

*Correspondence:

Zhaoyang Lu

luchaoy@163.com

Specialty section:

This article was submitted to

Structural Materials,

a section of the journal

Frontiers in Materials

Received: 15 December 2020

Accepted: 30 June 2021

Published: 21 December 2021

Citation:

Lu Z, Huang X and Huang J (2021) Role of Grain Size and Shape in

Superplasticity of Metals.

Front. Mater. 8:641928.

doi: 10.3389/fmats.2021.641928

\section{Role of Grain Size and Shape in Superplasticity of Metals}

\author{
Zhaoyang $\mathrm{Lu}^{1 *}$, Xuqiang Huang ${ }^{1}$ and Jingzhe Huang ${ }^{2}$ \\ ${ }^{1}$ School of Mechanical Engineering and Automation, Northeastern University, Shenyang, China, ${ }^{2}$ College of Resources and Civil \\ Engineering, Northeastern University, Shenyang, China
}

Superplasticity is characterized by an elongation to failure of $>300 \%$ and a measured strain rate sensitivity (SRS), close to 0.5 . The superplastic flow is controlled by diffusion processes; it requires the testing temperature of $0.5 T_{\mathrm{m}}$ or greater where $T_{\mathrm{m}}$ is the absolute melting temperature of metals. It is well established that a reduction in grain size improves the optimum superplastic response by lowering the deformation temperature and/or raising the strain rate. The low-temperature superplasticity (LTSP) is attractive for commercial superplastic forming, in view of lowering energy requirement, increasing life for conventional or cheaper forming dies, improving the surface quality of structural components, inhibiting quick grain growth and solute-loss from the surface layers, thus resulting in better post-forming mechanical properties. This paper will summarize the dependence of superplasticity on grain size and shape in various metallic materials, including ferrous and non-ferrous alloys, which has been considered as an effective strategy to enable the LTSP.

Keywords: superplasticity, grain refinement, grain size, low-temperature superplasticity, high-strain rate superplasticity, severe plastic deformation

\section{INTRODUCTION}

Superplasticity is the ability of metals to exhibit extremely large elongations of at least $300 \%$ prior to tensile failure, when deformation is imposed at relatively low strain rates (e.g., $\dot{\varepsilon}=10^{-3}$ to $10^{-5} \mathrm{~s}^{-1}$ ) and elevated temperatures $\left(T>0.5 T_{\mathrm{m}}\right)$, where $T_{\mathrm{m}}$ is the melting temperature of metals (Edington, 1982; Langdon, 1982). To date, superplasticity has been demonstrated in various metals, such as aluminium, copper, magnesium, zinc, titanium and iron base alloys etc., (Maehara and Langdon, 1990; Chokshi et al., 1993; Langdon, 2009; Reddy et al., 2017). Basically, superplastic flow may occur in two different ways (Maehara and Langdon, 1990; Langdon, 1991; Kaibbyshev, 2002): 1) In transformation superplasticity, the superplastic effect is induced by temperature cycling through phase transformation; 2) In structural superplasticity, the superplastic flow happens at constant temperature without phase transformation. As for structural superplasticity, several essential characteristics are involved. First, the initial microstructure is very fine (usually a few microns or less) and stable equiaxed grains upon tensile loading. Second, a strain-rate sensitivity exponent ( $m=\partial \ln \sigma / \partial \ln \dot{\varepsilon}$ ) ranges from 0.3 to 0.7 where $\sigma$ is the true stress and $\dot{\varepsilon}$ is the strain rate. Third, a dominant grain boundary sliding (GBS) mechanism occurs to facilitate isotropic deformation.

It is well established that a reduction in grain size improves the optimum superplastic response by lowering the deformation temperature to lower than $0.5 T_{\mathrm{m}}$ (low temperature superplasticity) and/or raising the strain rate higher than $10^{-3} s^{-}$(high strain rate superplasticity), which is beneficial for rapid superplastic forming of structural parts with nearly arbitrarily complex shapes at relatively low costs (Zhang et al., 2015). For achieving the superplasticity of metals, two groups of plastic 

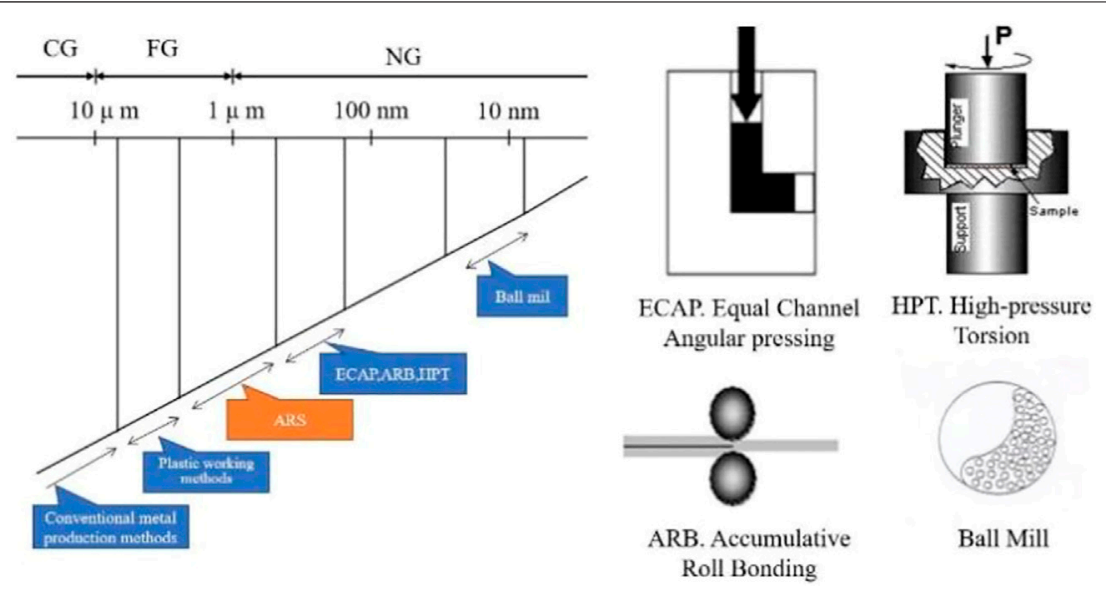

FIGURE 1 | Various techniques for production of UFG materials and the corresponding grain size levels. Note: CG-Coarse grains; FG-Fine grains; NG-Nano-sized grains.

deformation have been proposed to refine its microstructure down to ultrafine or nanoscale levels: One group is conventional plastic working processing (Tanaka et al., 2002; Tanaka et al., 2003; Xia et al., 2008), such as rolling, extrusion, forging and the other is severe plastic deformation (SPD) processing (Kumar et al., 2006; Chou et al., 2007; Yang et al., 2008; Kawasaki and Langdon, 2011) like equal-channel angular pressing (ECAP), high-pressure torsion (HPT) and friction stir welding (FSW), etc. By definition, nanoscaled materials have grain sizes of less than $100 \mathrm{~nm}$ and ultrafine-grained (UFG) materials have average grain sizes ranging from $100 \mathrm{~nm}$ to $1 \mu \mathrm{m}$. Various processing techniques to achieve ultra/nanograin refinement of metals are represented in Figure 1.

\section{SUPERPLASTICITY IN AL ALLOYS}

An early report documented the occurrence of superplasticity in Al-based alloys over strain rates covering several orders of magnitude (Higashi, 1994; Kawasaki and Langdon, 2007). It was found that samples processed by cold rolling (CR) were not superplastic because the imposed strains were insufficient to refine the size to the ultrafine or even smaller levels. Instead, it was necessary to use powder metallurgy (PM), physical vapor deposition or other techniques for the superplasticity of $\mathrm{Al}$ alloys achieved at low temperatures or high strain rates.

In contrast, the Al-alloys processed by ECAP were superplastic over at least a limited range of strain rates (Komura et al., 2001). It was found that several of the $\mathrm{Al}$ alloys exhibited exceptionally high elongations of $>2000 \%$, and the general trend of a bellshaped curve of total elongation against strain rate indicates three distinct flow regions where region II was superplastic at intermediate strain rates. It is also evident that superior elongations were achieved at high strain rates of no less than $10^{-2} \mathrm{~s}^{-1}$, thus implying the occurrence of high-strain-rate superplasticity.
Liu and Ma (2011) investigated the influence of grain size on superplastic behavior in the $\mathrm{Al}-\mathrm{Mg}$-Sc alloy processed by friction stir processing (FSP), as plotted in Figure 2. It was found that the medium-grained samples exhibited the largest elongation at the highest strain rate due to the optimum combination of grain size and its thermal stability. The superplasticity of Al-Mg-Sc alloy was mainly governed by the effective grain size (the grain size just before deformation) and its distribution just before tension rather than the initial grain size.

It was worth noting that the Al-6Mg-0.25Sc-0.1Zr alloy produced by asymmetric rolling (AsR) exhibited an average grain size of $\sim 1.5 \mu \mathrm{m}$, corresponding to an elongation of $\sim 3,200 \%$ at $773 \mathrm{~K}$ and $5 \times 10^{-2} \mathrm{~s}^{-1}$ (Xu et al., 2016). The superior superplasticity was ascribed to the ultrafine grains with a high fraction of high-angle grain boundaries (HAGBs) and disperse, coherent and nano-scaled $\mathrm{Al}_{3}(\mathrm{Sc}, \mathrm{Zr})$ particles.

\section{SUPERPLASTICITY IN TI ALLOYS}

It has been demonstrated that the $\mathrm{Ti}$ alloy processed by conventional SPD methods exhibited an average grain size of $75-400 \mathrm{~nm}$ and the LTSP was achieved at temperatures as low as 823-973 K (Kim et al., 1998; Salishchev et al., 2001; Ko et al., 2005; Ko et al., 2006; Matsumoto et al., 2013; Zhang et al., 2014a; Fu et al., 2015; Wu et al., 2016; Zhang et al., 2018a), as summarized in Table $\mathbf{1}$.

FSP is a novel solid-state processing technique for grain refinement and microstructural modification in view of the principle of friction stir welding (FSW) (Liu et al., 2009). Wu et al. (2016) reported that the friction stir welded Ti-6Al-4V alloy with a fully lamellar morphology had an excellent superplastic ductility of $\sim 728 \%$ at $1198 \mathrm{~K}$ and $3.0 \times 10^{-3} \mathrm{~s}^{-1}$, which reached $442 \%$ at $873 \mathrm{~K}$ and $3.0 \times 10^{-5} \mathrm{~s}^{-1}$.

In addition to the above-mentioned SPD methods, the initial microstructure also played an important role on ultra-grain 

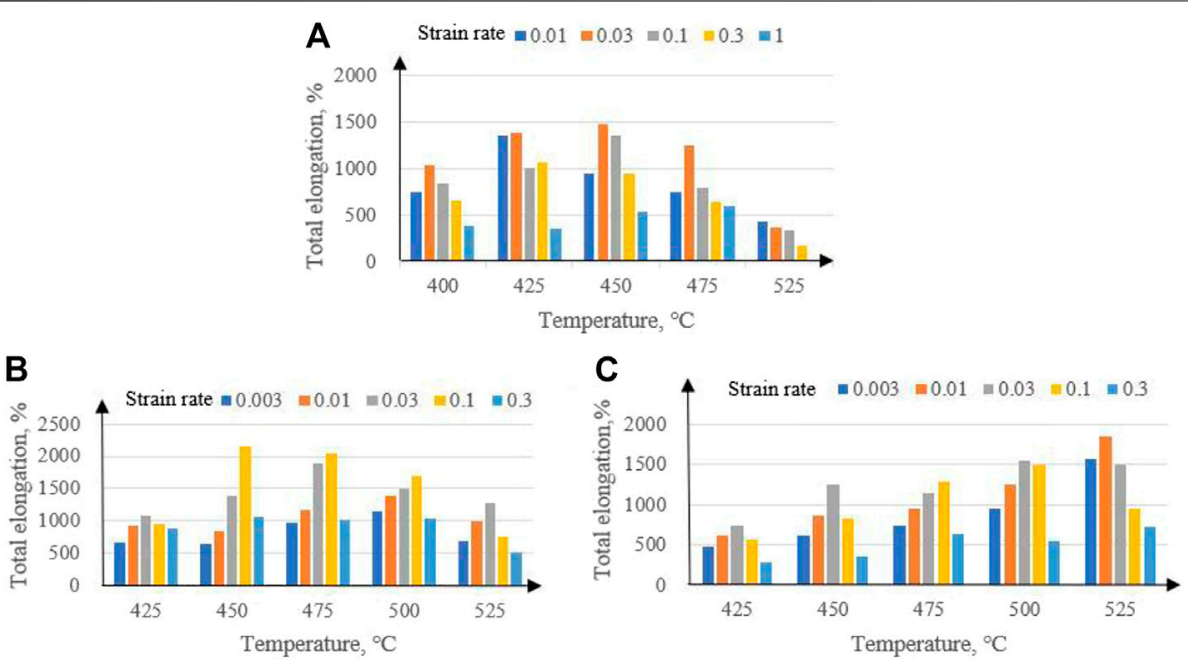

FIGURE 2 | Changes in total elongation with strain rate at different deformation temperatures in the Al-Mg-Sc alloy with grain sizes of $1.6 \mu \mathrm{m}$ (A), $2.6 \mu \mathrm{m}$ (B) and $2.9 \mu \mathrm{m}$ (C) (Liu and $\mathrm{Ma}, 2011)$.

TABLE 1 | Maximum elongations for the LTSP (below 973 K) in the SPDed Ti-6Al-4V alloy (Kim et al., 1998; Salishchev et al., 2001; Ko et al., 2005; Ko et al., 2006; Matsumoto et al., 2013; Zhang et al., 2014a; Fu et al., 2015; Wu et al., 2016; Zhang et al., 2018a).

\begin{tabular}{lcccc}
\hline Processing methods & $\mathbf{d}, \mathbf{n m}$ & $\boldsymbol{T}, \mathbf{K}$ & Strain rate, /s & Elongation, \% \\
\hline ECAP & 300 & $873-973$ & $873-923$ & $1.0 \times 10^{-4}-5.0 \times 10^{-4}$ \\
HPT & $75-300$ & $823-973$ & $5.0 \times 10^{-4}-1.0 \times 10^{-2}$ & $296-700$ \\
MAF & $135-400$ & $923-973$ & $5.0 \times 10^{-4}-7.0 \times 10^{-4}$ & $575-780$ \\
Hot rolling & $100-300$ & 923 & $1.0 \times 10^{-4}-1.0 \times 10^{-2}$ & $240-910$ \\
FSW & Fully lamellar & 873 & $3.0 \times 10^{-5}$ & $220-516$ \\
FSP & 510 & & $3.0 \times 10^{-4}$
\end{tabular}

Note: MAF, multiaxial forging.

TABLE 2 | Summary of experimental parameters and elongations in the Ti-6Al-4V alloy processed by HPT (Sergueeva et al., 2000; Sergueeva et al., 2002; Shahmir et al., 2018).

\begin{tabular}{lccc} 
Initial microstructure & $\boldsymbol{T}, \mathbf{K}$ & Strain rate, 1/s & Elongation, \% \\
\hline a+lamellar $(\alpha+\beta)$ & $923-998$ & $1.0 \times 10^{-3}-1.0 \times 10^{-2}$ & $504-676$ \\
$\alpha+75 \%$ lamellar $(\alpha+\beta)$ & 873 & $1.0 \times 10^{-4}-1.0 \times 10^{-2}$ & $540-790$ \\
Lamellar $(\alpha+\beta)$ & 973 & $1.0 \times 10^{-3}-1.0 \times 10^{-2}$ & $640-910$ \\
Martensitic & $923-973$ & $1.0 \times 10^{-4}-1.0 \times 10^{-2}$ & $410-690$
\end{tabular}

refinement of Ti-6Al-4V alloy and the resultant superplastic behavior. Wang and Langdon et al. (Wang and Langdon, 2013) demonstrated that the grain size of the HPT-processed Ti-6Al-4V alloy dropped with increasing the volume fraction of lamellar structure. Preliminary work (Fu et al., 2015; Zhang et al., $2018 \mathrm{~b}$ ) has also showed that as the fraction of lamellar structure reached $70 \%$, the grain size of the HPT-processed Ti-6Al-4V alloy was refined to $75 \pm 15 \mathrm{~nm}$, and an excellent LTSP of $\sim 780 \%$ was achieved at $873 \mathrm{~K}$ and $5.0 \times 10^{-4} \mathrm{~s}^{-1}$.

Yoon et al. (2015) investigated the effect of initial microstructure (as-received material, furnace-cooled, material and water-quenched material) on microstructural evolution in
Ti-6Al-4V alloy during FSW, and the equixaed microstructure with the smallest grain size in stir zone (SZ) center was formed for the initial martensitic microstructure. As demonstrated in Zhang et al. (2018a), an ultrafine microstructure consisting of $\alpha$ grains $(\sim 510 \mathrm{~nm})$ and a small amount of $\beta$ phase was successfully achieved in the FSPed Ti-6Al-4V alloy. The fraction of HAGBs with random crystallographic orientation was close to $90 \%$, revealing dynamic recrystallization was responsible for ultra-grain refinement during FSP. The LTSP of such an ultrafine microstructure was found in the temperature range of $773-923 \mathrm{~K}$ and strain rates from $1.0 \times 10^{-4} \mathrm{~s}^{-1}$ to $3.0 \times 10^{-3} \mathrm{~s}^{-1}$. Specifically, an exceptional LTSP of $1130 \%$ was achieved at $873 \mathrm{~K}$ and $3.0 \times 10^{-4} \mathrm{~s}^{-1}$.

Shahmir et al. (2018) examined the effect of initial microstructures, e.g. martensitic and lamellar on the superplasticity in the Ti-6Al-4V alloy processed by HPT. It was demonstrated that significant grain refinement was achieved under both conditions with grain sizes of $\sim 30$ and $\sim 40 \mathrm{~nm}$, respectively. The nanostructured samples exhibited excellent ductility at $923-1073 \mathrm{~K}$, including superplastic elongations at $973 \mathrm{~K}$ with maximum values of 815 and $690 \%$, respectively.

To provide a direct comparison with other published data on superplasticity in the Ti-6Al-4V alloy processed by HPT, Table 2 
TABLE 3 | Statistic results of chemical composition, thermomechanical processing and total elongation and deformation conditions for various ferrous superplastic alloys (Zhang et al., 2014b; Li et al., 2014; Cao et al., 2017; Han et al., 2017; Cao et al., 2018).

\begin{tabular}{|c|c|c|c|c|c|}
\hline Alloy & Composition (wt\%) & TMP & El, \% & $T_{\mathrm{d}},{ }^{\circ} \mathrm{C}$ & $\dot{\varepsilon}(\mathbf{1} / \mathbf{s})$ \\
\hline \multirow[t]{3}{*}{ Duplex stainless steel } & $25 \mathrm{Cr}-7 \mathrm{Ni}-3 \mathrm{Mo}$ & $\mathrm{HR}+\mathrm{CR}$ & 1700 & 1000 & $1.7 \times 10^{-2}$ \\
\hline & $22 \mathrm{Cr}-5 \mathrm{Ni}-3 \mathrm{Mo}$ & & 1510 & 980 & $3 \times 10^{-4}$ \\
\hline & $22 \mathrm{Cr}-5.4 \mathrm{Ni}-3.2 \mathrm{Mo}$ & $\mathrm{HR}+\mathrm{CR}$ & 1260 & 950 & $1.5 \times 10^{-3}$ \\
\hline \multirow[t]{7}{*}{ Ultra-high Carbon steel } & $1.3 \mathrm{C}-0.7 \mathrm{Mn}-0.1 \mathrm{Si}$ & $\mathrm{HR}+\mathrm{WR}$ & 700 & 630 & $1.3 \times 10^{-4}$ \\
\hline & $1.6 \mathrm{C}-0.7 \mathrm{Mn}-0.3 \mathrm{Si}$ & & 760 & 630 & $1.3 \times 10^{-4}$ \\
\hline & $1.9 \mathrm{C}-0.8 \mathrm{Mn}-0.3 \mathrm{Si}$ & & 380 & 650 & $1.7 \times 10^{-4}$ \\
\hline & $1.7 \mathrm{C}-1.5 \mathrm{Cr}-2.4 \mathrm{Al}-0.5 \mathrm{Mn}$ & multi-pass & 490 & 700 & $5 \times 10^{-4}$ \\
\hline & 1.3C-3.0Si-1.5Cr-0.5Mn & $\mathrm{HF}+\mathrm{WR}$ & 1300 & 850 & $1.7 \times 10^{-4}$ \\
\hline & 1.3C-2.3Mn-1.8Si-1.1Cr-1Al & $\mathrm{HR}+\mathrm{WR}$ & 720 & 780 & $2 \times 10^{-3}$ \\
\hline & 1.3C-2Mn-1.8Si-1.0Cr & $\mathrm{HR}+\mathrm{WQ}$ & 900 & 750 & $1 \times 10^{-4}$ \\
\hline \multirow[t]{3}{*}{ Medium Mn steel } & 0.05C-6.6Mn-2.3Al & $\mathrm{HR}+\mathrm{CR}$ & 1314 & 850 & $1 \times 10^{-3}$ \\
\hline & $0.1 \mathrm{C}-5 \mathrm{Mn}-2 \mathrm{Al}$ & $\mathrm{HR}+\mathrm{CR}$ & 1190 & 850 & $1 \times 10^{-3}$ \\
\hline & $0.1 \mathrm{C}-5 \mathrm{Mn}-2 \mathrm{Al}$ & $\mathrm{HR}$ & 340 & 800 & $1 \times 10^{-3}$ \\
\hline
\end{tabular}

Note: TMP, thermo-mechanical process; HR, hot rolling; WR, warm rolling; WQ, water quenching; CR, cold rolling.

summarizes the results reported under various parameters including initial microstructure and tensile elongation (Sergueeva et al., 2000; Sergueeva et al., 2002; Shahmir et al., 2018). All results showed high superplastic elongations but the maximum value of $815 \%$ was obtained. From this work, it was apparent that the initial grain size of the martensitic microstructure was smaller than the lamellar one and this would contribute to higher elongations, thus exhibiting exceptional superplasticity when compared with the lamellar microstructure.

\section{SUPERPLASTICITY IN FE ALLOYS}

The development of ferrous superplastic alloys allowed the mass production of complex parts at relatively low costs. For example, a promising superplasticity was found in some duplex stainless steels (DSS) and ultrahigh carbon steels (UCS) (Li et al., 2014; Zhang et al., 2014b). However, the requirements of high alloying contents ( $\mathrm{Cr}, \mathrm{Ni}$, and $\mathrm{Mo}$ ), high temperature or low strain rate hindered broad industrial applications for intricate parts. More recently, lean-alloyed Fe-Mn-Al-C steels (e.g., medium Mn steel, MMS) revealed an extraordinary superplasticity and they have significant potentials to be used as the next-generation automotive steels due to their high strength, excellent ductility, comparatively low density and material cost (Cao et al., 2017; Cao et al., 2018; Han et al., 2017). Table 3 summarized the chemical composition, thermomechanical processing and total elongation and deformation conditions for various ferrous superplastic alloys (Zhang et al., 2014b; Li et al., 2014; Cao et al., 2017; Han et al., 2017; Cao et al., 2018).
The superplastic MMSs were produced via either hot rolling or cold rolling followed by rapid cooling or inter-critical annealing, which evolved into a fully lath-typed or equiaxed ferrite and austenite $(\alpha+\gamma)$ duplex structure, respectively.

Han et al. (2017) investigated the superplasticity of Fe$6.6 \mathrm{Mn}-2.3 \mathrm{Al}$ cold rolled alloy. The tensile specimens were heated to $1123 \mathrm{~K}$, where the equilibrium volume fractions of both $\alpha$ and $\gamma$ phases were almost identical, held for $5 \mathrm{~min}$ and then tested at various initial strain rates. The process of lamella shearing happened during cold rolling, so that the equiaxed $(\alpha+\gamma)$ structure was quickly developed at elevated temperatures. Accordingly, a maximum elongation of $\sim 1314 \%$ was obtained at $1.0 \times 10^{-3} \mathrm{~s}^{-1}$. In contrast, the hot-rolled (HRed) lamellar structure induced high flow stresses at initial and steady states, making intergranular deformation occur more readily than GBS. This corresponds to a relatively low ductility of $\sim 340 \%$ at $800^{\circ} \mathrm{C}$ and $10^{-3} \mathrm{~s}^{-1}$ (Cao et al., 2018). The low temperature or high strain rate superplasticity of medium Mn steels is believed as a result of ultra-grain refinement and the slow diffusion of $\mathrm{Mn}$ and $\mathrm{Al}$ elements.

\section{GRAIN SIZE EFFECT ON SUPERPLASTICITY OF METALS}

Taking into account the grain size effect on high-temperature deformation, a model on superplastic deformation was proposed by Lou and Woo (2002), based on the theory of GBS and the theory of cavity growth, as follows: 


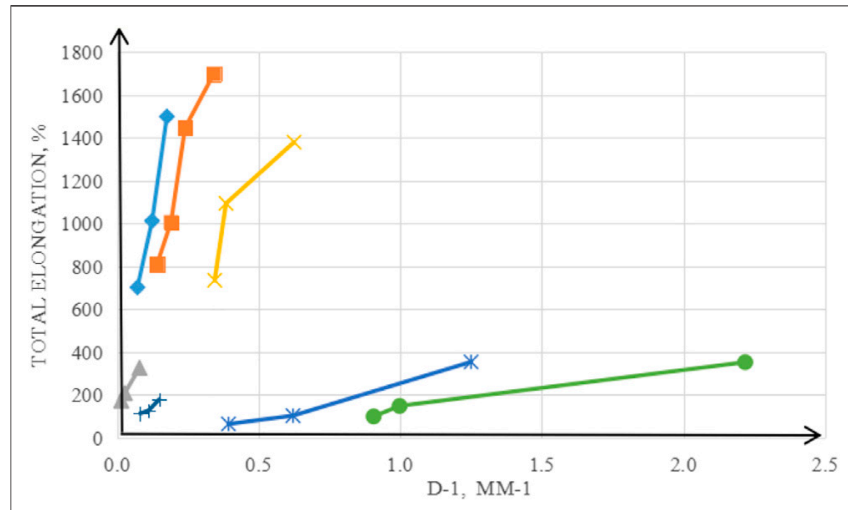

FIGURE 3 | Total elongation against inverse grain size for several superplastic materials. + Ti-6Al-4V deformed at $1200 \mathrm{~K}$ and $2.0 \times 10^{-4} \mathrm{~s}^{-1}$; Ternary brass deformed at $874 \mathrm{~K}$ and $1.67 \times 10^{-4} \mathrm{~s}^{-1} ; 7475 \mathrm{Al}$ alloy deformed at $789 \mathrm{~K}$ and $3.0 \times 10^{-4} \mathrm{~s}^{-1} ; \times$ Al-Mg-Sc alloy deformed at $698 \mathrm{~K}$ and 3.0 $\times 10^{-2} \mathrm{~s}^{-1} ; \times$ Al-14Ni-14Mn alloy deformed at $873 \mathrm{~K}$ and $1.0 \mathrm{~s}^{-1}$; $\bullet \mathrm{ZrO}$ dispersed Al deformed at $1727 \mathrm{~K}$ and $1.7 \times 10^{-4} \mathrm{~s}^{-1}$ (Liu and $\mathrm{Ma}, 2011$ ).

$$
r_{f}+\frac{B}{d}=C_{f} \exp \left(\varepsilon_{f}\right)
$$

where $\mathrm{B}$ is defined as:

$$
B=\left(\frac{45 \Omega \delta}{A b^{2}}-\frac{3 \gamma}{2 G}\right) \sqrt{\frac{A D_{g b} G b}{\dot{\varepsilon} k T}}
$$

where $\varepsilon_{f}$ is the total elongation to fracture, $C_{f}$ is an integrating constant, $r_{f}$ is the valid radius of cavity in fracture, $\Omega$ is the atomic volume, $\delta$ is the grain boundary width, $\gamma$ is the mean radius of cavity, $b$ is the Burgers vector, $D_{g b}$ is the grain boundary diffusivity, $\dot{\varepsilon}$ is the strain rate, $k$ is the Boltzman constant, $T$ is the absolute temperature, $A$ is the dimensionless constant and $G$ is the shear modulus.

The predictions of the model were compared with several published experimental data, as shown in Figure 3 (Liu and Ma, 2011). It was apparent that the total elongation increased with decreasing the grain size at various strain rates, i.e., the linear relationship between total elongation and $1 / d$ was kept in a strain rate range.

It is also well known that the contribution of GBS to the total macroscopic strain was estimated as being less than $70 \%$, so the remainder should be accommodated by other existing mechanisms. In some cases, high dislocation activity was still visible, not only in the vicinity of interphase boundaries, but also within grains even after failure, verifying that GBS was accommodated by dislocation slip or climb mechanisms. Regarding the dislocation accommodating mechanism, a common resemblance was found with the Rachinger GBS effect. The process was generally controlled by the equilibrium subgrain size parameter $\lambda$, which can be expressed as (Alabort et al., 2015):

$$
\lambda / b=\zeta(\sigma / \mu)^{-1}
$$

where $b$ is the magnitude of the Burgers' vector, $\sigma$ is the flow stress, $\mu$ is the shear modulus and $\zeta$ is a constant. Given that average grain size $(d)$ was constant, but $\lambda$ was related to the imposed stress and hence, as a function of deformation conditions.

In the case of phases whose size $d>\lambda$, intragranular dislocations would form subgrains and even evolve into new grains upon straining; whereas for phases whose size $d<\lambda$, intragranular dislocations traveled through the softer phase, in favor of intragranular slip.

It is well known that the flow stress of materials is a function of deformation temperature $T$, grain size $d$ and strain rate $\dot{\varepsilon}$ during superplastic deformation, as below (Zhu and Langdon, 2004):

$$
\dot{\varepsilon}=A \sigma^{n} d^{-p} D_{0} \exp [-Q /(R T)]
$$

where $\mathrm{A}$ is a constant, $n$ is an exponent related to the flow stress, $p$ is an exponent related to the grain size, $Q$ is an activation energy, $R$ is a gas constant. Thus, the strain rate can be found increase significantly when the grain size of materials is refined to a nanoscaled level. Langdon et al. reported that as the grain size is refined from $2 \mu \mathrm{m}$ to $200 \mathrm{~nm}$, the superplastic period is reduced from 20-30 $\mathrm{min}$ to $20-30 \mathrm{~s}$.

\section{CONCLUSION}

This work summarized the dependence of superplasticity of various metals on grain size and shape, including $\mathrm{Al}$ alloy, $\mathrm{Ti}$ alloy and ferrous alloy, etc. The smaller grains can contribute to lowering the deformation temperature and/or raising the strain rate, which is beneficial for rapid superplastic forming of parts with nearly arbitrarily complex shapes at relatively low costs. In comparison with severe plastic deformation, most of metals processed by conventional plastic deformation were not superplastic because the imposed strains were insufficient to refine the size to the ultrafine or even smaller levels. Meanwhile, the superplasticity of metals can be significantly improved as well by tailoring the initial microstructural parameters (grain shape, phase fraction, grain size distribution etc.), which is crucial for developing novel superplastic metals with commercial application potential.

\section{AUTHOR CONTRIBUTIONS}

$\mathrm{ZL}$ and $\mathrm{XH}$ have the idea to start new research works in superplasticity. JH was responsible for collecting information, drawing figures and editing tables. The article was written by $\mathrm{ZL}$ and revised by $\mathrm{XH}$. 


\section{REFERENCES}

Alabort, E., Putman, D., and Reed, R. C. (2015). Superplasticity in Ti-6Al-4V: Characterisation, Modelling and Applications. Acta Materialia 95, 428-442. doi:10.1016/j.actamat.2015.04.056

Cao, W. Q., Huang, C. X., Wang, C. Y., and Weng, Y. Q. (2017). Dynamic Reverse Phase Transformation Induced High-Strain-Rate Superplasticity in Low Carbon Low alloy Steels with Commercial Potential. Sci. Rep. 7, 9199. doi:10.1038/s41598-017-09493-7

Cao, Z. X., Wu, G. L., Sun, X. J., Wang, C., Ponge, D., and Cao, W. Q. (2018). Revealing the Superplastic Deformation Behaviors of Hot Rolled 0.10C5Mn2Al Steel with an Initial Martensitic Microstructure. Scripta Materialia 152, 27-30. doi:10.1016/j.scriptamat.2018.03.046

Chokshi, A., Mukherjee, A., and Langdon, T. (1993). Superplasticity in Advanced Materials. Mater. Sci. Eng. R: Rep. 10, 237-274. doi:10.1016/0927-796x(93)90009-r

Chou, C. Y., Lee, S. L., Lin, J. C., and Hsu, C. M. (2007). Effects of Cross-Channel Extrusion on the Microstructures and Superplasticity of a $\mathrm{Zn}-22$ wt.\% Al Eutectoid alloy. Scripta Materialia 57, 972-975.

Edington, J. W. (1982). Microstructural Aspects of Superplasticity. Mta 13, 703-715. doi:10.1007/BF02642384

Fu, J., Ding, H., Huang, Y., Zhang, W. J., and Langdon, T. G. (2015). Influence of Phase Volume Fraction on the Grain Refining of a Ti-6Al-4V alloy by HighPressure Torsion. J. Mater. Res. Technol. 4, 2-7. doi:10.1016/j.jmrt.2014.10.006

Han, J., Kang, S. H., Lee, S. J., Kawasaki, M., Lee, H. J., Ponge, D., et al. (2017). Superplasticity in a Lean Fe-Mn-Al Steel. Nat. Commun. 8, 751-757. doi:10.1038/s41467-017-00814-y

Higashi, K. (1994). Deformation Mechanisms of Positive Exponent Superplasticity in Advanced Aluminum Alloys with Nanoscale or Near-Nanoscale Grained Structures. Mater. Sci. Forum 170-172, 131-140. doi:10.4028/ www.scientific.net/msf.170-172.131

Kaibbyshev, O. A. (2002). Fundamental Aspects of Superplastic Deformation. Mater. Sci. Eng. A 324, 96-102. doi:10.1016/s0921-5093(01)01407-1

Kawasaki, M., and Langdon, T. G. (2007). Principles of Superplasticity in UltrafineGrained Materials. J. Mater. Sci. 42, 1782-1796. doi:10.1007/s10853-0060954-2

Kawasaki, M., and Langdon, T. G. (2011), Developing Superplasticity and a Deformation Mechanism Map for the $\mathrm{Zn}-\mathrm{Al}$ Eutectoid alloy Processed by High-Pressure Torsion. Mater. Sci. Eng. A. 528, 6140-6145. doi:10.1016/ j.msea.2011.04.053

Kim, J. S., Chang, Y. W., and Lee, C. S. (1998). Quantitative Analysis on Boundary Sliding and its Accommodation Mode during Superplastic Deformation of Two-phase Ti-6Al-4V alloy. Metallurgical Mater. Trans. A 29, 217-226. doi:10.1007/s11661-998-0174-2

Ko, Y. G., Kim, W. G., Lee, C. S., and Shin, D. H. (2005). Microstructural Influence on Low-Temperature Superplasticity of Ultrafine-Grained Ti-6Al-4V alloy. Mater. Sci. Eng. A 410-411, 156-159. doi:10.1016/j.msea.2005.08.080

Ko, Y. G., Lee, C. S., Shin, D. H., and Semiatin, S. L. (2006). Low-temperature Superplasticity of ultra-fine-grained Ti-6Al-4V Processed by Equal-Channel Angular Pressing. Metallurgical Mater. Trans. A 37, 381-391. doi:10.1007/ s11661-006-0008-z

Komura, S., Horita, Z., Furukawa, M., Nemoto, M., and Langdon, T. G. (2001). An Evaluation of the Flow Behaviour during High Strain Rate Superplasticity in an Al-Mg-Sc alloy. Metallurgical Mater. Trans. A 32, 707-714. doi:10.1007/ s11661-001-0087-9

Kumar, P., Xu, C., and Langdon, T. G. (2006). Mechanical Characteristics of a $\mathrm{Zn}-22 \% \mathrm{Al}$ alloy Processed to Very High Strains by ECAP. Mater. Sci. Eng. A 429, 324-328. doi:10.1016/j.msea.2006.05.044

Langdon, T. G. (2009). Seventy-five Years of Superplasticity: Historic Developments and New Opportunities. J. Mater. Sci. 44, 5998-6010. doi:10.1007/s10853-009-3780-5

Langdon, T. G. (1982). The Mechanical Properties of Superplastic Materials. Mta 13, 689-701. doi:10.1007/BF02642383

Langdon, T. G. (1991). The Physics of Superplastic Deformation. Mater. Sci. Eng. A 137, 1-11. doi:10.1016/0921-5093(91)90312-b

Li, S. X., Ren, X. P., Ji, X., and Gui, Y. Y. (2014). Effects of Microstructure Changes on the Superplasticity of 2205 Duplex Stainless Steel. Mater. Des. 55, 146-151. doi:10.1016/j.matdes.2013.09.042
Liu, F. C., Ma, Z. Y., and Chen, L. Q. (2009). Low-temperature Superplasticity of Al-Mg-Sc alloy Produced by Friction Stir Processing. Scripta Materialia 60, 968-971. doi:10.1016/j.scriptamat.2009.02.021

Liu, F. C., and Ma, Z. Y. (2011). Superplasticity Governed by Effective Grain Size and its Distribution in fine-grained Aluminum Alloys. Mater. Sci. Eng. A 510, 548-558. doi:10.1016/j.msea.2011.10.018

Lou, T. P., and Woo, K. D. (2002). Influence of Grain Size on Fracture of Superplasticity. Mater. Lett. 52, 374-377. doi:10.1016/s0167-577x(01)00426-8 Maehara, Y., and Langdon, T. G. (1990). Superplasticity of Steels and Ferrous Alloys. Mater. Sci. Eng. A 128, 1-13. doi:10.1016/0921-5093(90)90090-P

Matsumoto, H., Yoshida, K., Lee, S. H., Ono, Y., and Chib, A. (2013). Ti-6Al-4V alloy with an Ultrafine-Grained Microstructure Exhibiting LowTemperature-High-Strain-Rate Superplasticity. Mater. Lett. 98, 209-212. doi:10.1016/j.matlet.2013.02.033

Reddy, S. R., Bapari, S., Bhattacharjee, P. P., and Chokshi, A. H. (2017). Superplasticlike Flow in a fine-grained Equiatomic CoCrFeMnNi High-Entropy alloy. Mater. Res. Lett. 5 (6), 408-414. doi:10.1080/21663831.2017.1305460

Salishchev, G. A., MGaleyev, R., Valiakhmetov, O. R., Safiullin, O. N., Lutfullin, R. Y., Senkov, O. N., et al. (2001). Development of Ti-6Al-4V Sheet with Low Temperature Superplastic Properties. J. Mater. Process. Technol. 116, 265-268. doi:10.1016/S0924-0136(01)01037-8

Sergueeva, A. V., Stolyarov, V. V., Valiev, R. Z., and Mukherjee, A. K. (2000). Enhanced Superplasticiy in a Ti-6Al-4V alloy Processed by Severe Plastic Deformation. Scripta Materialia 43, 819-824. doi:10.1016/S1359-6462(00)00496-6

Sergueeva, A. V., Stolyarov, V. V., Valiev, R. Z., and Mukherjee, A. K. (2002). Superplastic Behaviour of Ultrafine-Grained Ti-6A1-4V Alloys. Mater. Sci. Eng. A 323, 318-325. doi:10.1016/S0921-5093(01)01384-3

Shahmir, H., Naghdi, F., Pereira, P. H., Huang, Y., and Langdon, T. G. (2018). Factors Influencing Superplasticity in the Ti-6Al-4V alloy Processed by High Pressure Torsion. Mater. Sci. Eng. A 718, 198-206. doi:10.1016/ j.msea.2018.01.091

Tanaka, T., Makii, K., Kushibe, A., and Higashi, K. (2002). Room Temperature Deformation Behavior of $\mathrm{Zn}-22$ mass\%Al alloy with Nanocrystalline Structure. Mater. Trans. 43, 2449-2454. doi:10.2320/matertrans.43.2449

Tanaka, T., Makii, K., Kushibe, A., Kohzu, M., and Higashi, K. (2003). Capability of Superplastic Forming in the Seismic Device Using Zn-22Al Eutectoid alloy. Scripta Materialia 49, 361-366. doi:10.1016/S1359-6462(03)00328-2

Wang, Y. C., and Langdon, T. G. (2013). Influence of Phase Volume Fractions on the Processing of a Ti-6Al-4V alloy by High-Pressure Torsion. Mater. Sci. Eng. A 559, 861-867. doi:10.1016/j.msea.2012.09.034

Wu, L. H., Xiao, B. L., and Ma, Z. Y. (2016). Achieving superior Low-Temperature Superplasticity for Lamellar Microstructure in Nugget of a Friction Stir Welded Ti6Al-4V Joint. Scripta Materialia 122, 26-30. doi:10.1016/j.scriptamat.2016.05.020

Xia, S. H., Wang, J., Wang, J. T., and Liu, J. Q. (2008). Improvement of RoomTemperature Superplasticity in Zn-22 wt.\%Al alloy. Mater. Sci. Eng. A 493, 111-115. doi:10.1016/j.msea.2007.07.100

Xu, G. F., Cao, X. W., Duan, Y. L., Peng, X. Y., Deng, Y., and Yin, Z. M. (2016). Achieving High Strain Rate Superplasticity of an Al-Mg-Sc-Zr alloy by a New Asymmetrical Rolling Technology. Mater. Sci. Eng. A 672, 98-107. doi:10.1016/ j.msea.2011.10.01810.1016/j.msea.2016.06.070

Yang, C.-F., Pan, J.-H., and Chuang, M.-C. (2008). Achieving High Strain Rate Superplasticity via Severe Plastic Deformation Processing. J. Mater. Sci. 43, 6260-6266. doi:10.1007/s10853-008-2909-2

Yoon, S., Ueji, R., and Fujii, H. (2015). Effect of Initial Microstructure on Ti-6Al-4V Joint by Friction Stir Welding. Mater. Des. 88, 1269-1276. doi:10.1016/j.matdes.2015.09.128

Zhang, H., Pradeep, K. G., Mandal, S., Ponge, D., Choi, P., Tasan, C. C., et al. (2014). Enhanced Superplasticity in an Al-Alloyed Multicomponent $\mathrm{Mn}-\mathrm{Si}-\mathrm{Cr}-\mathrm{C}$ Steel. Acta Materialia 63, 232-244. doi:10.1016/j.actamat.2013.10.034

Zhang, N. X., Kawasaki, M., Huang, Y., and Langdon, T. G. (2015). Influence of Grain Size on Superplastic Properties of a Two-phase Pb-Sn alloy Processed by Severe Plastic Deformation. J. Mater. Metall. 14, 255-304.

Zhang, T. Y., Liu, Y., Sanders, D. G., Liu, B., Zhang, W. D., and Zhou, C. X. (2014). Development of fine-grain Size Titanium 6Al-4V alloy Sheet Material for Low Temperature Superplastic Forming. Mater. Sci. Eng. A 608, 265-272. doi:10.1016/j.msea.2014.04.098

Zhang, W. J., Ding, H., Cai, M. H., Yang, W. J., and Li, J. Z. (2018). Ultra-grain Refinement and Enhanced Low-Temperature Superplasticity in a Friction 
Stir-Processed Ti-6Al-4V alloy. Mater. Sci. Eng. A 727, 90-96. doi:10.1016/ j.msea.2018.03.009

Zhang, W. J., Ding, H., Pereira, P. H., Huang, Y., and Langdon, T. G. (2018). Grain Refinement and Superplastic Flow in a Fully Lamellar Ti-6Al-4V alloy Processed by High-Pressure Torsion. Mater. Sci. Eng. A 732, 398-405. doi:10.1016/ j.msea.2018.07.010

Zhu, Y. T., and Langdon, T. G. (2004). The Fundamentals of Nanostructured Materials Processed by Severe Plastic Deformatioin. JOM 56, 58-63. doi:10.1007/s11837-004-0294-0

Conflict of Interest: The authors declare that the research was conducted in the absence of any commercial or financial relationships that could be construed as a potential conflict of interest.
Publisher's Note: All claims expressed in this article are solely those of the authors and do not necessarily represent those of their affiliated organizations, or those of the publisher, the editors and the reviewers. Any product that may be evaluated in this article, or claim that may be made by its manufacturer, is not guaranteed or endorsed by the publisher.

Copyright $\odot 2021 \mathrm{Lu}$, Huang and Huang. This is an open-access article distributed under the terms of the Creative Commons Attribution License (CC BY). The use, distribution or reproduction in other forums is permitted, provided the original author(s) and the copyright owner(s) are credited and that the original publication in this journal is cited, in accordance with accepted academic practice. No use, distribution or reproduction is permitted which does not comply with these terms. 